\title{
Corrigendum
}

\section{Corrigendum to "HDR Brachytherapy in the Management of High-Risk Prostate Cancer”}

\author{
Susan Masson, ${ }^{1}$ Raj Persad, ${ }^{2}$ and Amit Bahl ${ }^{1}$ \\ ${ }^{1}$ Bristol Haematology and Oncology Centre, University Hospitals Bristol NHS Foundation Trust, Bristol BS2 8ED, UK \\ ${ }^{2}$ Department of Urology, University Hospitals Bristol NHS Foundation Trust, Bristol BS2 8HW, UK \\ Correspondence should be addressed to Raj Persad; raj.persad@uhbristol.nhs.uk \\ Received 18 September 2016; Accepted 26 September 2016 \\ Copyright (c) 2016 Susan Masson et al. This is an open access article distributed under the Creative Commons Attribution License, \\ which permits unrestricted use, distribution, and reproduction in any medium, provided the original work is properly cited.
}

In the article titled "HDR Brachytherapy in the Management of High-Risk Prostate Cancer" [1], there was an error in Table 2, which should be corrected as follows:
For Hoskin et al. [32], the dose should read $35.75 \mathrm{~Gy}$ in 13 fractions EBRT, not 55 Gy in 20 fractions EBRT.

TABLE 2: Estimated equivalent doses (2Gy per fraction) for published HDR schedules using the EQD2 formula, assuming $\alpha / \beta$ for prostate cancer of 1.5 Gy [19].

\begin{tabular}{lcc}
\hline Author & Schedule & EQD2 prostate $(\alpha / \beta 1.5$ Gy) \\
\hline Galalae et al. [36] & 50 Gy WPRT, 40 Gy Prostate EBRT, 2 fractions HDR (9 Gy per fraction) & 94 Gy \\
Åström et al. [30] & 50 Gy in 25 fractions EBRT, 2 fractions HDR (10 Gy per fraction) & 115.7 Gy \\
Martinez et al. [28] & 46 Gy in 23 fractions EBRT, 2 fractions HDR (11.5 Gy per fraction) & 131.4 Gy \\
Hoskin et al. [32] & 35.75 Gy in 13 fractions EBRT, 2 fractions HDR (8.5 Gy per fraction) & 92.0 Gy \\
\hline
\end{tabular}

\section{References}

[1] S. Masson, R. Persad, and A. Bahl, "HDR brachytherapy in the management of high-risk prostate cancer," Advances in Urology, vol. 2012, Article ID 980841, 6 pages, 2012. 


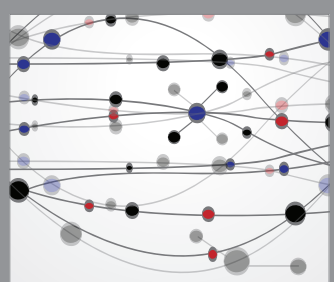

The Scientific World Journal
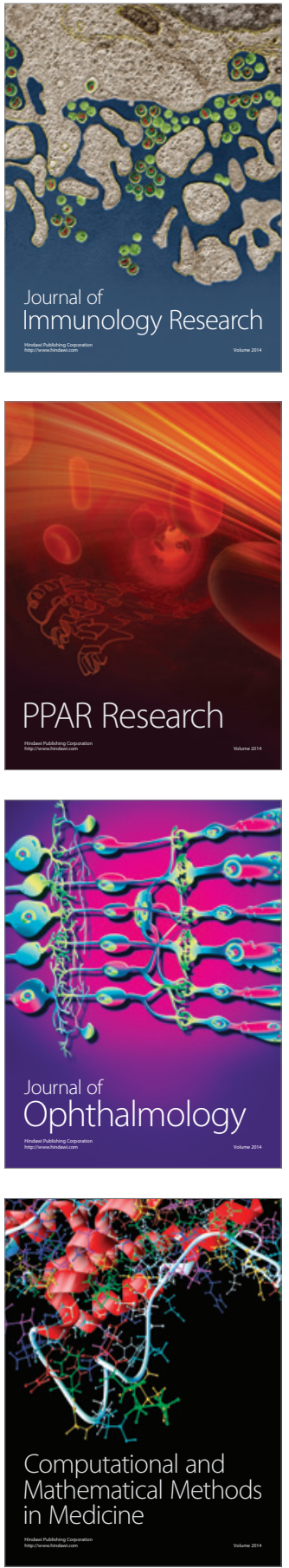

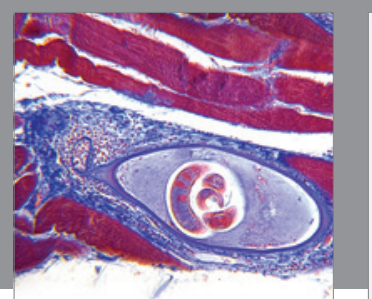

Gastroenterology Research and Practice

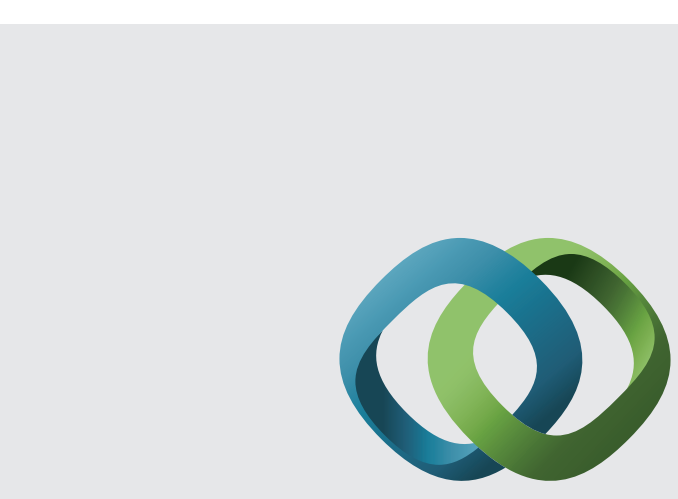

\section{Hindawi}

Submit your manuscripts at

http://www.hindawi.com
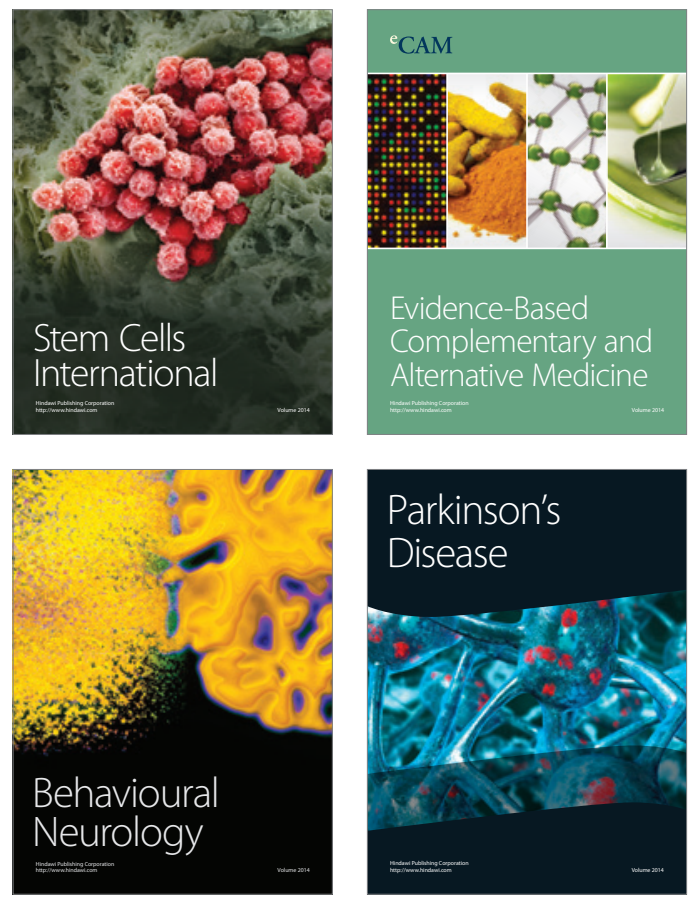
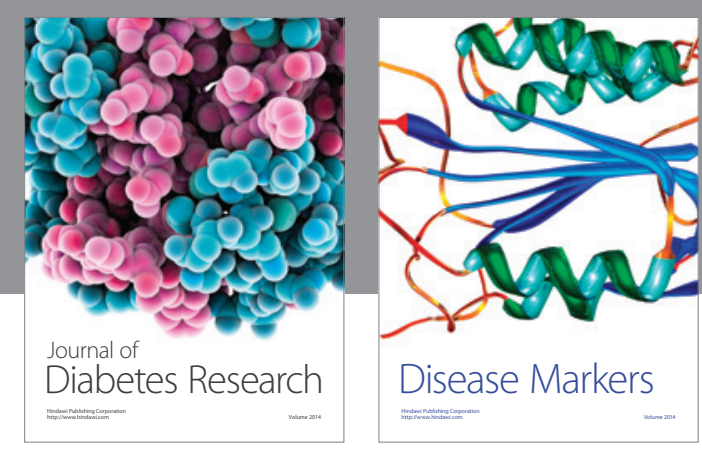

Disease Markers
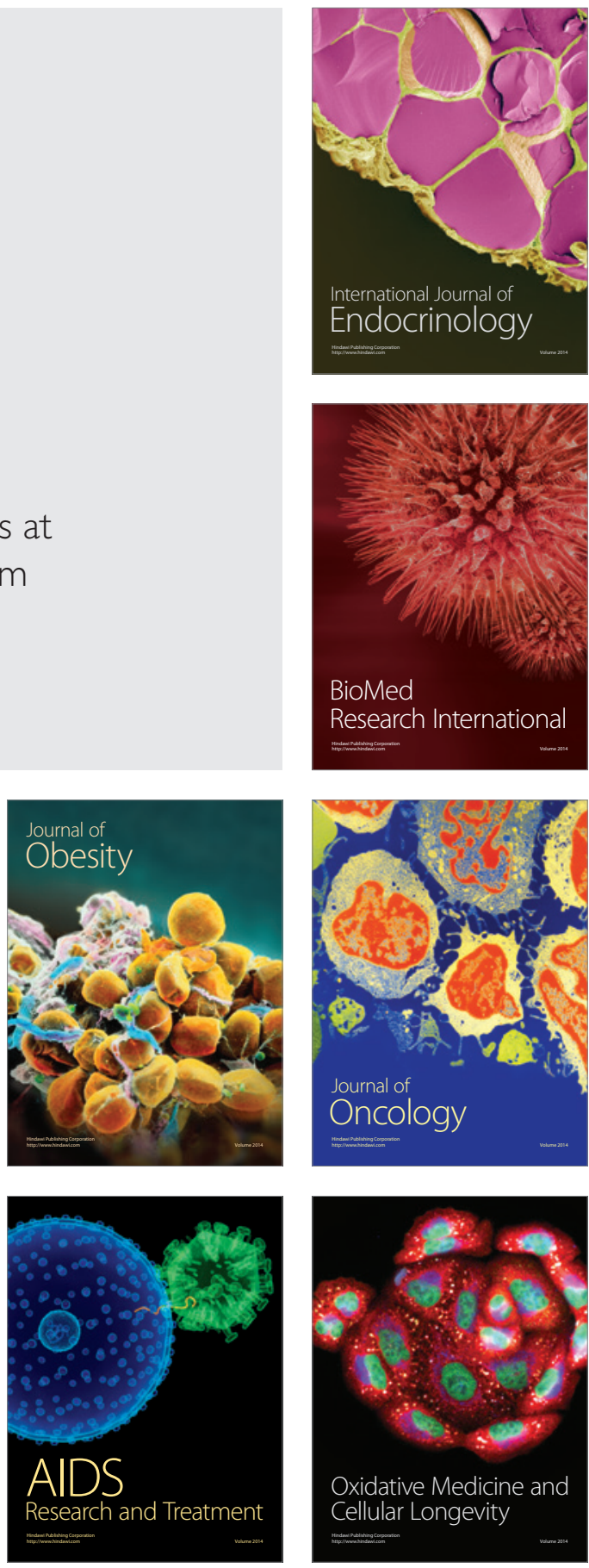